\title{
Prognostic significance of transcription factors FOXA1 and GATA-3 in ductal carcinoma in situ in terms of recurrence and estrogen receptor status
}

\author{
Mamatha Chivukula ${ }^{1}$, Jennifer Picarsic ${ }^{2}$, Gautam Bulusu' ${ }^{1}$ Adam Brufsky², Gretchen Ahrendt ${ }^{3}$, Gloria Carter \\ ${ }^{I}$ Department of Pathology, Mills-Peninsula Helath Services, Dorothy Schneider Breast Cancer Center, Burlingame, CA 94050 , USA. \\ ${ }^{2}$ Department of Pathology, Magee-Women's Hospital of UPMC, Pittsburgh, PA 15213, USA. \\ ${ }^{3}$ Department of Oncology, University of Pittsburgh Cancer Institute, Pittsburgh, PA 15232, USA. \\ ${ }^{4}$ Department of Surgery, Magee-Women's Hospital of UPMC, Pittsburgh, PA 15213, USA.
}

Correspondence to: Dr. Mamatha Chivukula, Department of Pathology, Mills-Peninsula Helath Services, Dorothy Schneider Breast Cancer Center, Burlingame, CA 94050, USA. E-mail: mchivukula@ppmgpath.com

\section{A B S T R A C T}

Aim: The aim was to analyze the expression of novel biological transcription markers, forkhead-box A1 (FOXA1), GATA binding protein 3 (GATA-3), and established markers such as Ki-67 (MIB-1) and human epidermal growth factor receptor 2 (HER2) in estrogen receptor (ER(+)) and ER(-) ductal carcinoma in situ (DCIS) patients with/without recurrence. Methods: Two hundred and ninety-one cases of DCIS were retrieved from our pathology database, with complete data available for 219 cases. The follow-up period is from 1988 to 2009. Recurrence is defined in terms of DCIS or invasive carcinoma (IC). No recurrence was seen in $88 \%(196 / 219)$ of cases; $12 \%$ (26/219) had a recurrence (IC: 13, DCIS: 13). We are reporting the results of biological marker expression in terms of recurrence and ER status. Results: Our study demonstrates strong expression of GATA-3 in the ER(+) DCIS in recurrence and nonrecurrence groups similar to previously described in IC. A reduced expression of GATA-3 was observed in ER(-) recurrence and nonrecurrence groups. A strong HER2 protein expression, as well as high proliferation index, was seen in recurrence group (DCIS and IC). FOXA1 expression is reduced across the groups though not statistically significant. Conclusion: This is the first study to analyze novel transcription markers FOXA1 and GATA-3 in DCIS. Further work needs to be done on a larger cohort of DCIS cases with recurrence to better understand, which variables are best able to predict recurrence and guide therapy decision strategies. Maintenance of FOXA1 and GATA-3 expression in ER(-) DCIS may offer new promising targets for therapy in future.

Key words: Ductal carcinoma in situ, estrogen receptor, forkhead-box A1, GATA binding protein 3

\section{Introduction}

Ductal carcinoma in situ (DCIS) is a heterogeneous pre-invasive carcinoma and has become a significant proportion of screen-detected breast malignancies in North American and Western Europe, since the onset of wide-spread screening mammography nearly two decades ago. ${ }^{[1,2]}$ Unlike invasive breast carcinoma (IC), DCIS is a more heterogeneous malignancy without clear prognostic indicators for recurrence, defined as either recurrent DCIS or IC. While the Van Nuys Prognostic Index, based on histopathologic indicators (high nuclear grade, necrosis, margin width, and size) has been used clinically for predicting recurrence, there has been no good biomarker(s) that predicts outcome in DCIS. ${ }^{[3]}$ Furthermore, whereas IC has been classified into distinct molecular subtypes (luminal A/B, human epidermal growth factor

\begin{tabular}{|l|l|}
\hline \multicolumn{2}{|c|}{ Access this article online } \\
\hline Quick Response Code: & Website: \\
\hline & www.jcmtjournal.com \\
\cline { 2 - 2 } & \\
\hline
\end{tabular}

receptor 2 (HER2)-like, basal-like), which confer prognostic clinical significance, to date few studies have attempted to classify DCIS into similar molecular-based subtypes. ${ }^{[4-6]}$ There is emerging evidence on limited data to suggest cDNA microarray, gene-expression profiles can segregate DCIS into similar distinct molecular subtypes as in $\mathrm{IC}^{\cdot[4]}$ however, a significant proportion of DCIS shows tumor heterogeneity ${ }^{[4,5,7]}$ making it difficult to stratify DCIS cases into a single subtype, and thus subsequently diminishing the prognostic significance of these molecular subtypes, as compared to IC.

Estrogen receptor (ER) status has been the leading candidate biomarker in DCIS, as it plays a key role in development and influences hormonal treatment in IC patients. Absence of ER was shown to be one of the significant predictors of recurrence in $\mathrm{IC} .^{[8]}$ In addition, it is well-known that about $30 \%$ of $\mathrm{ER}(+)$ tumors are not hormone responsive, and about $5-15 \%$ of ER(-) tumors are responsive to anti-estrogen therapy. ${ }^{[9]}$ However, the two broad groups of IC namely $\mathrm{ER}(+)$ and ER(-), are yet to be well understood in DCIS.

Recently, various research groups have looked at the functional interaction between the forkhead-box A1 (FOXA1) winged helix transcription factor and GATA binding protein 3 (GATA-3), a zinc finger transcription 
factor, in their role in suppressing ER-dependent breast cancer cell growth and tumor genesis and maintenance of breast luminal-cell differentiation in vivo. Their use as prognostic clinical biomarkers has recently been studied in IC but not in DCIS. ${ }^{[10-13]}$ FOXA1, originally called the hepatocyte nuclear factor $3 \alpha$, is a ubiquitous transcription factor expressed in liver, breast, prostate, lung, colon, and pancreas that has both activator and repressor activity. As an activator, FOXA1 has the unique ability to bind to its target sites via altering chromatin structure facilitating ER $\alpha$ binding and thus promoting gene expression. ${ }^{[14,15]}$ FOXA1 may also act as a growth inhibitor via binding and activation of the p27 promoter, located within the BRCA1-responsive element, ${ }^{[16,17]}$ thus plays a critical role in suppressing ER-dependent breast cancer cell growth and tumor genesis in vivo. ${ }^{[17]}$

GATA-3, a member of the zinc finger DNA binding proteins, was originally discovered in its role in T-lymphoid development into Th2 cells. ${ }^{[18]}$ In the breast, GATA-3 was initially discovered to be associated with ER expression in breast carcinomas ${ }^{[19]}$ and has subsequently been demonstrated in vivo to be highly expressed in the mammary luminal epithelial cells, responsible for both development and maintenance of luminal cells fate. ${ }^{[20,21]}$ Like FOXA1, GATA-3 is also promising biomarker and the complex relationship between ER $\alpha$, FOXA1, and GATA-3 is being better understood in order to refine the hormone-responsive phenotype in IC, which will help both with therapy decision making and better prediction of clinical outcomes. ${ }^{[21-23]}$ GATA-3 has been identified as an upstream promoter of FOXA1 transcription. ${ }^{[20]}$ FOXA1 has been shown to be responsible for expression of at least $50 \%$ of ER $\alpha$ regulated genes ${ }^{[24-26]}$ and thus has been proposed as the link between GATA-3 and ER $\alpha .{ }^{[21]}$ GATA-3 genes are involved with induction of FOXA1 expression, with increased activity in $\operatorname{ER}(+)$ carcinoma; therefore, the highest expression of FOXA1 in IC should be seen in association with both GATA-3 and ER $\alpha$ expression, ${ }^{[27]}$ which has been seen in IC. ${ }^{[12]}$ However, this relationship has not yet been categorized in DCIS. The specific aim of this study is to analyze the expression for the first time in DCIS of novel biological transcription markers FOXA1, GATA-3, along with established markers MIB-1 (Ki-67) and HER2-neu in $\mathrm{ER}(+)$ and ER(-) groups of DCIS. As it has been shown in IC, we will investigate if there is a similar association between FOXA1/GATA-3 with ER $\alpha$ in DCIS. The secondary goal is to define an expression profile of FOXA1, GATA-3 and other biomarkers that could predict recurrence in these DCIS groups and further stratify low versus high-risk patients and impact treatment decisions.

\section{Methods}

\section{Patients}

In our retrospective study, we identified 2,434 women diagnosed with DCIS from 1988 to 2009 from the tumor registry data. Paraffin-embedded blocks and hematoxylin and eosin (HE) slides of 291 patients with initial DCIS were retrieved. Complete demographic data, menopause state, hormone therapy use, family history, prior history of pregnancy, mammography report (mass, calcifications), surgical treatment and adjuvant therapy, along with pathologic data were reviewed. All patients had undergone a core needle biopsy or needle localization excision biopsy for initial diagnosis. Two hundred and nineteen cases who had complete follow-up, glass slides and tumor bocks were chosen for the study. Recurrence was defined as DCIS or invasive breast cancer in the same breast 1-year or more after the initial diagnosis of DCIS. Nonrecurrence DCIS group included patients who had DCIS or microinvasion (invasion $\leq 1 \mathrm{~mm}$ ) or invasive cancer that was subsequently diagnosed at the time of complete excision.

\section{Procedure}

The project was approved by the University of Pittsburgh Institutional Review Board. All cases were reviewed by two pathologists with confirmation of nuclear grade, as described by conventional features observed on the HE slide. All other pathologic features were obtained from the original report. The tumor size measurement was assessed either by size from microscopic or gross description or as an estimation based on tumor volume from number of slides involved per total slides. Margins were considered clear (negative) defined as no link on the tumor and positive if less than $1 \mathrm{~mm}$. Table 1 shows complete clinical, radiological and pathologic characteristics in relation to recurrence.

Immunohistochemistry (IHC) was performed on the selected paraffin-embedded tumor block of the index DCIS lesion using the following biomarkers GATA-3, FOXA1, ER, progesterone receptor (PR), Ki-67 and HER2.

Predilute rabbit monoclonal antibodies directed against ER alpha (SP1), PR (1E2) and HER2 (4B5) were purchased from Ventana Medical Systems Inc., Tucson, AZ, USA (VMSI). The manufacturer's recommended protocols were followed, utilizing CC1 for antigen unmasking, and iVIEW/DAB (Ventana Medical Systems, Inc.) for detecting the antigen-antibody complex and a biotin block to inhibit nonspecific staining of endogenous biotin. Mouse monoclonal anti-GATA-3 (L50-823), purchased from BD Biosciences was diluted 1:300 and shared the same protocol parameters as the previous mentioned. FOXA1 protein was detected using a goat polyclonal antibody from Santa Cruz. Slides were pretreated in a steamer in Target Retrieval Solution, $\mathrm{pH} 6.0$ (Dako) at $95{ }^{\circ} \mathrm{C}$ for $20 \mathrm{~min}$, then cooled at room temperature. Slides were then incubated with anti-FOXA1 (1:400) followed by Goat Immpress/DAB polymer for detection (Vector Labs) [Table 2]. 
Table 1: Clinical, radiological and pathologic characteristics in relation to each DCIS case

\begin{tabular}{|c|c|c|c|c|}
\hline \multirow[t]{2}{*}{$\overline{\text { Characteristics }}$} & \multicolumn{2}{|c|}{$\begin{array}{c}\text { Nonrecurrence } \\
\text { group }\end{array}$} & \multicolumn{2}{|c|}{$\begin{array}{l}\text { Recurrence } \\
\text { group }\end{array}$} \\
\hline & DCIS & $\begin{array}{c}\text { DCIS } \\
\text { with/IC }\end{array}$ & DCIS & IC \\
\hline \multicolumn{5}{|l|}{ Radiology } \\
\hline Calcifications & 126 & 41 & 1 & 10 \\
\hline Mass & 16 & 7 & 2 & 2 \\
\hline Nipple discharge & 5 & 1 & 0 & 1 \\
\hline \multicolumn{5}{|l|}{ Chemotherapy } \\
\hline Positive & 1 & 9 & 0 & 0 \\
\hline Negative & 6 & 35 & 0 & 0 \\
\hline NA & 140 & 2 & 0 & 0 \\
\hline \multicolumn{5}{|l|}{ Radiation therapy } \\
\hline Positive & 82 & 29 & 45 & 20 \\
\hline Negative & 44 & 14 & 2 & 1 \\
\hline NA & 21 & 6 & 0 & 0 \\
\hline \multicolumn{5}{|l|}{ Surgery } \\
\hline Segmental (bilateral) & 4 & 1 & 0 & 1 \\
\hline Segmental (unilateral) & 106 & 25 & 22 & 12 \\
\hline TM (bilateral) & 17 & 7 & 10 & 8 \\
\hline TM (unilateral) & 28 & 13 & 15 & 0 \\
\hline \multicolumn{5}{|l|}{ Nuclear grade } \\
\hline 1 & 20 & 2 & 0 & 1 \\
\hline 2 & 57 & 34 & 26 & 3 \\
\hline 3 & 70 & 13 & 21 & 17 \\
\hline Size $(\mathrm{cm})$ & $\begin{array}{c}1.8 \\
(0.3-10)\end{array}$ & $\begin{array}{c}2.0 \\
(0.35-9.0)\end{array}$ & $\begin{array}{c}1.6 \\
(0.3-9.0)\end{array}$ & $\begin{array}{c}0.8 \\
(0.2-2.4\end{array}$ \\
\hline \multicolumn{5}{|l|}{ Focality } \\
\hline Unifocal & 76 & 37 & 2 & 8 \\
\hline Multifocal & 71 & 12 & 40 & 13 \\
\hline \multicolumn{5}{|l|}{ Lymph node biopsy } \\
\hline Done & 56 & 46 & 2 & 1 \\
\hline Not done & 91 & 3 & 45 & 20 \\
\hline \multicolumn{5}{|l|}{ Margin status } \\
\hline Positive & 45 & 12 & 3 & 14 \\
\hline Negative & 102 & 34 & 42 & 7 \\
\hline NA & 0 & 0 & 2 & 0 \\
\hline Total & 147 & 46 & 13 & 13 \\
\hline
\end{tabular}

DCIS: Ductal carcinoma in situ; IC: Invasive carcinoma; TM: Total mastectomy; Segmental: Segmental mastectomy; NA: Not available

Table 2: Antibodies used for immunohistochemical characterization of ductal carcinoma in situ

\begin{tabular}{llll}
\hline Antibody & Clone & Dilution & Source \\
\hline FOXA1 & $\begin{array}{l}\text { Goat } \\
\text { polyclonal }\end{array}$ & $1: 400$ & $\begin{array}{l}\text { Santa Cruz Biotechnology, } \\
\text { Inc.; Santa Cruz, CA, USA }\end{array}$ \\
GATA-3 & L50-823 & $1: 300$ & BD Biosciences, USA \\
HER2-neu & 485 & Predilute & Ventana; Tucson, AZ, USA \\
Ki-67 & $30-9$ & Predilute & Ventana; Tucson, AZ, USA \\
ER & SP1 & Predilute & Ventana; Tucson, AZ, USA \\
PR & 1E2 & Predilute & Ventana; Tucson, AZ, USA \\
\hline
\end{tabular}

FOXA1: Forkhead-box A1; GATA-3: GATA binding protein 3; HER2: Human epidermal growth factor receptor 2; ER: Estrogen receptor; PR: Progesterone receptor

Positive and negative control tissues were used for assessment of each marker. FOXA1, GATA-3, ER, PR, and nuclear stains were evaluated with a cumulative "H score" based on proportionality score and intensity scores (0-10: negative; 11-150: low; 151-250: intermediate; 250-300: high). A $10 \%$ or more "nuclear" staining of the tumor cells was considered "positive." The proliferation marker, Ki-67/MIB-1, was given a nuclear proliferation index (1-10\%: low; 11-25\%: intermediate; 26-50\%: high; > 51\%: very high); HER2 a membrane stain, was interpreted as per routine guidelines for IC $(0 / 1+$ negative, $2+$ weakly positive, $3+$ strongly positive) [Figure 1].

\section{Statistical analysis}

Four risk groups based on ER expression were defined separately for subsequent invasive cancer/DCIS based on the risk associated with clinical/histopathologic characteristics and biomarker expression. Groups were defined by combining biomarker expression that had similar strength associations and level of risk for subsequent tumor events. We used Fisher's exact test to determine the dependence between clinical outcomes in the $\mathrm{ER}(+)$ and $\mathrm{ER}(-)$ groups with and without recurrence for each biomarker and calculation of $P$ value. We also examined combinations of these biomarkers that were found as individual markers in univariate analyses to be statistically significantly associated with invasive cancer and/or DCIS or were previously shown to have a biological basis for association with subsequent tumors after a DCIS diagnosis or were previously reported to be associated with breast cancer survival.

\section{Results}

Of the total 219 patients selected for study, with a median follow-up of 4.5 years (range: 1-21 years); $88 \%$ (196/219) developed no recurrence. In $12 \%(26 / 219)$ patients who developed subsequent recurrence; $6 \%(13 / 26)$ recurred as IC; $6 \%(13 / 26)$ as DCIS. In the nonrecurrence group, 67\% (146/196) were pure DCIS on both biopsy and final excision; $26 \%(46 / 196)$ cases had subsequent IC associated with DCIS on final excision. The IC associated with DCIS were all ductal carcinomas. Their overall nuclear grade is 1,2 and 3, which constitution ratio is $4 \%(2 / 46)$, $87 \%$ (40/46), 9\% (4/46), respectively. The mean tumor size in the DCIS with subsequent IC group was $0.4 \mathrm{~cm}$ (range $0.25-3.5 \mathrm{~cm})$. Seventy percent $(136 / 196)$ of nonrecurrence group and 92\% (24/26) of recurrence group were treated with breast-conserving surgery alone. Fifty-eight percent $(111 / 196)$ in the nonrecurrence group and $77 \%(20 / 26)$ in the recurrence group were treated with radiation therapy. In both the groups, negative (clear) surgical margins, defined as no ink on the tumor on final excision, were obtained in $70 \%(136 / 196)$ nonrecurrence group and 62\% (16/26) cases of the recurrence group. The mean tumor size in DCIS with recurrence as IC group was $1.5 \mathrm{~cm}$ (mean $0.1-4.5 \mathrm{~cm})$. Several morphologic characteristics were 
reviewed, and none showed statistical significance with an increased risk of subsequent DCIS, although the index DCIS lesions with high nuclear grade, which had positive or uncertain margins showed a higher rate of recurrence.

We are reporting the results of biological markers expression in terms of recurrence and ER status. ER(-) DCIS with and without recurrence had lower expression of GATA-3 $(P<0.05)$ than ER $(+)$ cases. A strong HER2 overexpression $(P<0.05)$ and higher proliferation index of Ki-67 $(P<0.05)$ were seen in ER(-) group. FOXA1 as an individual biomarker expressed in $\mathrm{ER}(+)$ and ER(-) groups was not statistically significant. Nearly all ER(-) cases retained expression of FOXA1 and GATA-3. Overall, cases with recurrence demonstrated the greater percentage of ER(-), HER2 overexpression, and high proliferation compared to nonrecurrence cases [Table 3 and Figure 2].

\section{Discussion}

This is one of the first studies to analyze novel transcription factors in DCIS patients, and we show that FOXA1 and GATA-3 expression is strongly seen in both ER(-) and $\mathrm{ER}(+)$ DCIS groups. We observed that strong expression of FOXA1 and GATA-3, low/intermediate Ki-67, and

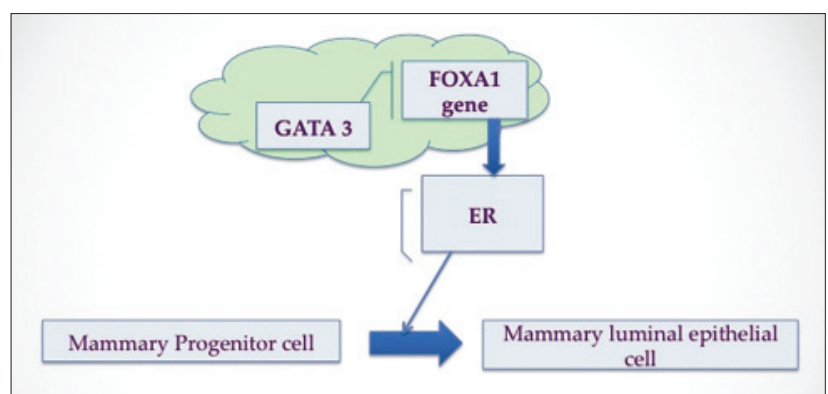

Figure 1: Pathway describing the role of GATA-3 and FOXA1 in development and maintenance of mammary luminal cells. GATA-3 promoter of FOXA1 transcription which in turn is responsible for expression of ERa regulated genes. FOXA1: Forkhead-box A1; GATA-3: GATA binding protein 3 low/absent HER2-neu expression were characteristically seen in our ER(+) DCIS groups, similar to previously described in IC. ${ }^{[23]}$ While there is a statistically significant lower expression of GATA-3 in the ER(-) cases, nearly all maintained expression. A significant number of ER(-) DCIS cases showed FOXA1 expression. FOXA1 and GATA-3 transcription factors have been shown to correlate highly with the luminal A molecular subtype of IC. ${ }^{[10,11]}$ Within the luminal, a subtype of IC, it has been shown that FOXA1 ${ }^{[10,11]}$ and GATA-3 ${ }^{[22,24]}$ can sub-classify patients into a low and high-risk groups based on their strong expression. FOXA1 via its actions on the p27 promoter, ${ }^{[16,17]}$ is thought to maintain IC in a less proliferative state, with a decreased metastatic potential, ${ }^{[10-13]}$ while GATA-3 is important in the maintenance of tumor differentiation and suppression of metastatic potential. ${ }^{[21]}$ Therefore, it is not surprising that these transcription factors are highly expressed in DCIS as well, which by definition is an in situ (noninvasive) carcinoma.

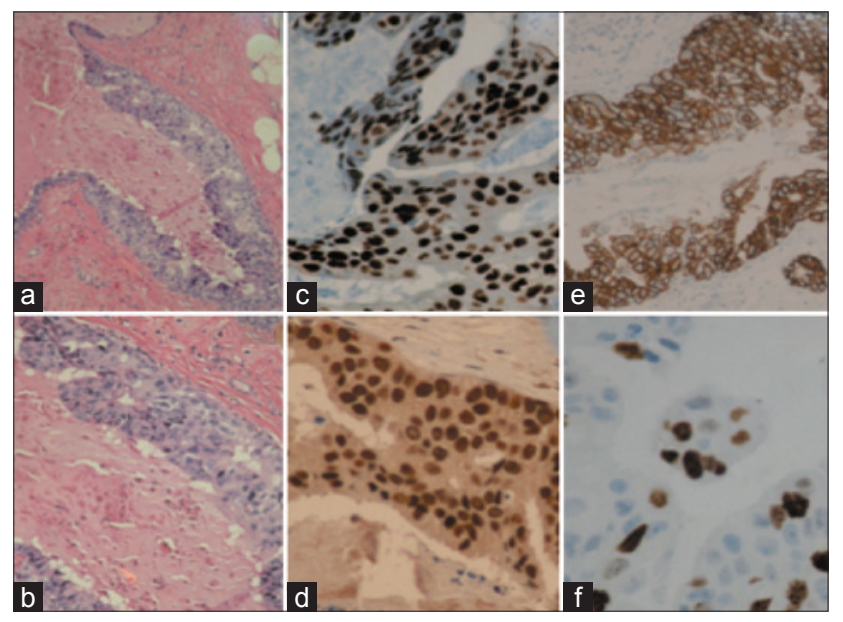

Figure 2: Representative high grade ductal carcinoma in situ case (×40). (a and b) Hematoxylin and eosin stain; (c) GATA-3 expression (H score 162); (d) FOXA1 expression (H score 180); (e) human epidermal growth factor receptor 2/neu expression (immunohistochemistry score 3+); (f) Ki-67 expression (15\% proliferation rate). FOXA1: Forkhead-box A1; GATA-3: GATA binding protein 3

Table 3: Biomarker score in DCIS, stratified by hormone status and follow up outcome

\begin{tabular}{|c|c|c|c|c|c|c|c|c|c|c|c|c|}
\hline & $n(\%)$ & ER score & PR score & $P$ & GATA-3 & $P$ & FOXA1 & $P$ & HER2 & $P$ & Ki 67 & $P$ \\
\hline \multicolumn{13}{|c|}{ Recurrence DCIS } \\
\hline ER(-) & $6(46)$ & 1 & 1 & $0.019^{*}$ & 150 & 0.12 & 169 & 0.50 & 3 & $0.032 *$ & 35 & 0.11 \\
\hline $\mathrm{ER}(+)$ & $7(54)$ & 150 & 96 & & 190 & & 169 & & 1 & & 19 & \\
\hline \multicolumn{13}{|c|}{ Recurrence IC } \\
\hline ER(-) & $4(31)$ & 0 & 0 & $0.034^{*}$ & 165 & $0.021 *$ & 192 & 0.08 & 2 & 0.14 & 16 & 0.31 \\
\hline $\mathrm{ER}(+)$ & $9(69)$ & 182 & 75 & & 212 & & 216 & & 1 & & 20 & \\
\hline \multicolumn{13}{|c|}{ No recurrence DCIS } \\
\hline ER(-) & 34 (23) & 1 & 6 & $<0.001 *$ & 158 & $<0.001^{*}$ & 178 & $0.026^{*}$ & 3 & $<0.001 *$ & 29 & $<0.001^{*}$ \\
\hline $\mathrm{ER}(+)$ & $113(77)$ & 208 & 96 & & 204 & & 195 & & 1 & & 16 & \\
\hline \multicolumn{13}{|c|}{ No recurrence IC } \\
\hline ER(-) & $11(24)$ & 0 & 1 & $<0.001 *$ & 172 & $0.019^{*}$ & 223 & 0.255 & 3 & $<0.001^{*}$ & 47 & $<0.01^{*}$ \\
\hline $\mathrm{ER}(+)$ & $35(76)$ & 197 & 92 & & 228 & & 211 & & 2 & & 26 & \\
\hline
\end{tabular}

$\mathrm{ER}(-)$ represents $\mathrm{H}$ score $<10, \mathrm{ER}(+)$ represents $\mathrm{H}$ score $>10 .{ }^{*} P$ value with statistical significance. ER: Estrogen receptor; PR: Progesterone receptor; DCIS: Ductal carcinoma in situ; IC: Invasive carcinoma; FOXA1: Forkhead-box A1; GATA-3: GATA binding protein 3 
There was a trend toward lower GATA-3 expression in all groups of ER(-) DCIS compared to the ER(+) cases. There was not a significant difference in the recurrence group; however, our numbers are low and may be lacking statistical power to draw meaningful conclusions. Others have shown that in IC with low/absent expression of GATA-3 expression, there is an association with absence of hormone receptor expression for ER/PR, overexpression of HER2, and most significantly, shorter disease-free survival. ${ }^{[24]}$

For ER(+) luminal type-A invasive cancers, FOXA1 is a significant predictor of cancer survival. ${ }^{[10,11]}$ Interestingly, high FOXA1 expression in ER(-) IC has also been shown to confer a lower risk of recurrence, ${ }^{[12]}$ while loss of GATA-3 expression in ER(+) is associated with a higher rate of recurrence and/or metastasis. ${ }^{[24]}$ These data suggest that FOXA1 and GATA-3 expression in IC has a complex relationship with ER. These novel transcription factors appear to be important prognostic biomarkers associated with a well-differentiated state. These data help explain why our DCIS cases maintained such high expression of GATA-3 and FOXA1 even within the ER(-) group. It would be of importance to know the difference in the level of expression between cases at recurrence and at diagnosis, index to see if there is an incremental decrease in transcription factor expression at recurrence. In this pilot study, we were not able to perform this comparison.

Others have shown in DCIS that the loss of ER expression along with HER2-neu overexpression is a predictor of recurrence. ${ }^{[8,28]}$ Similarly, we saw this pattern in our cases, with a higher percentage of ER(-), HER2-neu positive cases in the recurrent group compared to the nonrecurrent group. It is our hypothesis that with greater statistical power and optimization of our antibody titers that we may see a small but significantly lower expression in FOXA1 and GATA-3 in recurrent cases, as was seen with greater loss of ER expression in this group.

Further work needs to be done on a larger cohort of DCIS cases with recurrence to understand better which variables are best able to predict recurrence and guide therapy decision strategies. Our study compared two novel biomarkers, along with established biomarkers and other important histopathological, clinical, and treatment factors, in a novel prediction model, to determine which factors best predict recurrence in DCIS. The maintenance of FOXA1 and GATA-3 expression in ER(-) DCIS needs to be evaluated further, as these transcription factors may offer new promising targets for therapy.

\section{Acknowledgments}

The authors would like to thank Kim McManus (Q IHC) for the technical (IHC) assistance.

\section{References}

1. Ernster VL, Barclay J, Kerlikowske K, Grady D, Henderson C. Incidence of and treatment for ductal carcinoma in situ of the breast. JAMA 1996;275:913-8.

2. Ernster VL, Ballard-Barbash R, Barlow WE, Zheng Y, Weaver DL, Cutter G, Yankaskas BC, Rosenberg R, Carney PA, Kerlikowske K, Taplin SH, Urban N, Geller BM. Detection of ductal carcinoma in situ in women undergoing screening mammography. J Natl Cancer Inst 2002;94:1546-54.

3. Altintas S, Lambein K, Huizing MT, Braems G, Asjoe FT, Hellemans H, Van Marck E, Weyler J, Praet M, Van den Broecke R, Vermorken JB, Tjalma WA. Prognostic significance of oncogenic markers in ductal carcinoma in situ of the breast: a clinicopathologic study. Breast $J$ 2009;15:120-32.

4. Allred DC, Wu Y, Mao S, Nagtegaal ID, Lee S, Perou CM, Mohsin SK, O'Connell P, Tsimelzon A, Medina D. Ductal carcinoma in situ and the emergence of diversity during breast cancer evolution. Clin Cancer Res 2008;14:370-8.

5. Tang P, Wang X, Schiffhauer L, Wang J, Bourne P, Yang Q, Quinn A, Hajdu SI. Relationship between nuclear grade of ductal carcinoma in situ and cell origin markers. Ann Clin Lab Sci 2006;36:16-22.

6. Livasy CA, Perou CM, Karaca G, Cowan DW, Maia D, Jackson S, Tse CK, Nyante S, Millikan RC. Identification of a basal-like subtype of breast ductal carcinoma in situ. Hum Pathol 2007;38:197-204.

7. Tamimi RM, Baer HJ, Marotti J, Galan M, Galaburda L, Fu Y, Deitz AC, Connolly JL, Schnitt SJ, Colditz GA, Collins LC. Comparison of molecular phenotypes of ductal carcinoma in situ and invasive breast cancer. Breast Cancer Res 2008;10:404-13.

8. Provenzano E, Hopper JL, Giles GG, Marr G, Venter DJ, Armes JE. Biological markers that predict clinical recurrence in ductal carcinoma in situ of the breast. Eur J Cancer 2003;39:622-30.

9. Jordan VC, Wolf MF, Mirecki DM, Whitford DA, Welshons WV. Hormone receptor assays: clinical usefulness in the management of carcinoma of the breast. Crit Rev Clin Lab Sci 1988;26:97-152.

10. Badve S, Turbin D, Thorat MA, Morimiya A, Nielsen TO, Perou CM, Dunn S, Huntsman DG, Nakshatri H. FOXA1 expression in breast cancer - correlation with luminal subtype A and survival. Clin Cancer Res 2007;13:4415-21.

11. Thorat MA, Marchio C, Morimiya A, Savage K, Nakshatri H, Reis-Filho JS, Badve S. Forkhead box A1 expression in breast cancer is associated with luminal subtype and good prognosis. $J$ Clin Pathol 2008;61:327-32.

12. Albergaria A, Paredes J, Sousa B, Milanezi F, Carneiro V, Bastos J, Costa S, Vieira D, Lopes N, Lam EW, Lunet N, Schmitt F. Expression of FOXA1 and GATA-3 in breast cancer: the prognostic significance in hormone receptor-negative tumours. Breast Cancer Res 2009;11:R40.

13. Habashy HO, Powe DG, Rakha EA, Ball G, Paish C, Gee J, Nicholson RI, Ellis IO. Forkhead-box A1 (FOXA1) expression in breast cancer and its prognostic significance. Eur $J$ Cancer 2008;44:1541-51.

14. Lacroix M, Leclercq G. About GATA-3, HNF3A, and XBP1, three genes co-expressed with the oestrogen receptor-alpha gene (ESR1) in breast cancer. Mol Cell Endocrinol 2004;219:1-7.

15. Kourzarides T. Chromatin modifications and their function. Cell 2007;128:693-705.

16. Williamson EA, Wolf I, O'Kelly J, Bose S, Tanosaki S, Koeffler HP. BRCA1 and FOXA1 proteins coregulate the 
expression of the cell cycle-dependent kinase inhibitor p27(Kip1). Oncogene 2006;25:1391-9.

17. Wolf I, Bose S, Williamson EA, Miller CW, Karlan BY, Koeffler HP. FOXA1: growth inhibitor and a favorable prognostic factor in human breast cancer. Int $J$ Cancer 2007;120:1013-22.

18. Ho IC, Vorhees P, Marin N, Oakley BK, Tsai SF, Orkin SH, Leiden JM. Human GATA3: a lineage-restricted transcription factor that regulates the expression of the $\mathrm{T}$ cell receptor gene. EMBO J 1991;10:1187-92.

19. Hoch RV, Thompson DA, Baker RJ, Weigel RJ. GATA-3 is expressed in association with estrogen receptor in breast cancer. Int J Cancer 1999;84:122-8.

20. Kouros-Mehr H, Slorach EM, Sternlicht MD, Werb Z. GATA-3 maintains the differentiation of the luminal cell fate in the mammary gland. Cell 2006;127:1041-55.

21. Kouros-Mehr H, Kim JW, Bechis SK, Werb Z. GATA-3 and the regulation of the mammary luminal cell fate. Curr Opin Cell Biol 2008;20:164-70.

22. Jacquemier J, Charafe-Jauffret E, Monville F, Esterni B, Extra JM, Houvenaeghel G, Xerri L, Bertucci F, Birnbaum D. Association of GATA3, P53, Ki67 status and vascular peritumoral invasion are strongly prognostic in luminal breast cancer. Breast Cancer Res 2009;11:R23.

23. Mehra R, Varambally S, Ding L, Shen R, Sabel MS, Ghosh D, Chinnaiyan AM, Kleer CG. Identification of GATA3 as a breast cancer prognostic marker by global gene expression meta-analysis. Cancer Res 2005;65:11259-64.

24. Carroll JS, Liu XS, Brodsky AS, Li W, Meyer CA, Szary AJ, Eeckhoute J, Shao W, Hestermann EV, Geistlinger TR, Fox EA, Silver PA, Brown M. Chromosome-wide mapping of estrogen receptor binding reveals long-range regulation requiring the forkhead protein FoxA1. Cell 2005;122:33-43.

25. Carroll JS, Brown M. Estrogen receptor target gene: an evolving concept. Mol Endocrinol 2006;20:1707-14.

26. Laganiere J, Deblois G, Lefebvre C, Bataille AR, Robert F, Giguère V. From the Cover: Location analysis of estrogen receptor alpha target promoters reveals that FOXA1 defines a domain of the estrogen response. Proc Natl Acad Sci U S A 2005;102:11651-6.

27. Nakshatri H, Badve S. FOXA1 in breast cancer. Expert Rev Mol Med 2009;11:e8.

28. Keeple J, Henry-Tillman RS, Klimberg VS, Layeeque R, Siegel E, Westbrook K, Korourian S. The receptor expression pattern in ductal carcinoma in situ predicts recurrence. Am J Surg 2006;192:68-71.

How to cite this article: Chivukula M, Picarsic J, Bulusu G, Brufsky A, Ahrendt G, Carter G. Prognostic significance of transcription factors FOXA1 and GATA-3 in ductal carcinoma in situ in terms of recurrence and estrogen receptor status. J Cancer Metastasis Treat 2015;1:84-9.

Received: 18-12-2014; Accepted: 21-04-2015.

Source of Support: Nil, Conflict of Interest: None declared 